\section{Distanciamento social, uso de máscaras e higienização das mãos entre participantes do Estudo Longitudinal da Saúde dos Idosos Brasileiros: iniciativa ELSI-COVID-19}

\author{
Social distancing, use of face masks and hand \\ washing among participants in the Brazilian \\ Longitudinal Study of Aging: the ELSI-COVID-19 \\ initiative
}

\section{Distanciamiento social, uso de mascarillas e higienización de las manos entre participantes del Estudio Brasileño Longitudinal del Envejecimiento: iniciativa ELSI-COVID-19}

Maria Fernanda Lima-Costa 1,2 Juliana Vaz de Melo Mambrini 1 Fabiola Bof de Andrade 1 Sérgio William Viana Peixoto 1 James Macinko 3

doi: 10.1590/0102-311X00193920

\title{
Resumo
}

O objetivo do estudo foi examinar a prevalência do distanciamento social, do uso de máscaras e da higienização das mãos ao sair de casa entre adultos brasileiros com 50 anos ou mais de idade. Foram utilizados dados de 6.149 entrevistas telefônicas, conduzidas entre 26 de maio e 8 junho de 2020 dentre os participantes do Estudo Longitudinal da Saúde dos Idosos Brasileiros (ELSI-Brasil). O distanciamento social foi definido por não ter saído de casa nos últimos 7 dias. Somente 32,8\% dos participantes do estudo não saíram de casa no período considerado, 36,3\% saíram entre 1 e 2 vezes, 15,2\% entre 3 a 5 vezes e 15,7\% saíram todos os dias. As principais razões para sair de casa foram comprar remédios ou alimentos $(74,2 \%)$, trabalhar $(25,1 \%)$, pagar contas (24,5\%), atendimento dem saúde (10,5\%), fazer exercícios $(6,2 \%)$ e encontrar familiares ou amigos (8,8\%). Entre os que sairam de casa, 97,3\% usaram sempre máscaras faciais e 97,3\% sempre higienizaram as mãos. As mulheres sairam menos de casa que os homens. Esses saíram com mais frequência para trabalhar e fazer exercícios. Elas saíram mais para atendimento em saúde. Os homens (odds ratio - $O R=1,84)$ aqueles com escolaridade mais alta $(O R=$ 1,48 e 1,95 para 5-8 e 9 anos, respectivamente) e os residentes em áreas urbanas $(O R=1,54)$ saíram mais para realizar atividades essenciais, independentemente da idade e de outros fatores relevantes. Os resultados mostram baixa adesão ao distanciamento social, mas altas prevalências nos usos de máscaras e higienização das mãos.

Coronavirus; COVID-19; Máscaras; Isolamento Social; Envelhecimento

\author{
Correspondência \\ M. F. Lima-Costa \\ Núcleo de Estudos em Saúde Pública e Envelhecimento, Instituto \\ René Rachou, Fundação Oswaldo Cruz. \\ Av. Augusto de Lima 1715, 6o andar, sala 614, Belo Horizonte, \\ MG 30190-003, Brasil. \\ lima.costa@fiocruz.br \\ 1 Instituto René Rachou, Fundação Oswaldo Cruz, \\ Belo Horizonte, Brasil. \\ 2 Programa de Pós-graduação em Saúde Púbica, \\ Universidade Federal de Minas Gerais, Belo Horizonte, Brasil. \\ 3 University of California, Los Angeles, U.S.A.
}




\section{Introdução}

Globalmente, a epidemia do coronavírus 2 (SARS-CoV-2) é o maior desafio atual para a Saúde Pública. A epidemia iniciou em Wuhan, na China, em dezembro de 2019, e rapidamente se espalhou por diferentes países e continentes, levando a Organização Mundial da Saúde a considerá-la uma pandemia em 11 de março de 2020 1. Uma semana depois, o Brasil reconheceu a existência da transmissão comunitária do vírus 2 e, desde então, os casos vêm crescendo exponencialmente e sem sinais de arrefecimento. No final de junho, o Brasil ocupava a 2a posição no mundo, tanto em número de casos confirmados (1,37 milhão de casos) quanto em número de óbitos (58.314 óbitos), ficando atrás apenas dos Estados Unidos 3.

O coronavírus 2 é transmitido por meio de secreções provenientes do sistema respiratório. Ações governamentais, como o fechamento total (lockdown), proibição de eventos públicos e aglomerações, fechamento de escolas e encorajamento ao distanciamento social, para evitar contato com portadores sintomáticos e não sintomáticos do vírus, são recomendadas para conter ou diminuir a transmissão $4,5,6,7,8,9$. De forma complementar, são recomendadas medidas de proteção individual, como isolamento voluntário, uso de máscaras faciais e higienização das mãos 5,10,11,12.

A epidemia do coronavírus 2 no Brasil ocorre em um cenário de disputas políticas, que levaram à troca de dois ministros da saúde em um curto espaço de tempo. Embora o país tenha estabelecido precocemente linhas gerais para o enfrentamento da epidemia (Lei no 13.979, de 6 de fevereiro de 2020 13), ela tem sido alvo de narrativas desencontradas, com negação ou diminuição da sua gravidade pela autoridade central 14 . Na ausência de orientação clara e oportuna no nível federal, a implementação de medidas de controle e prevenção vem sido assumida por governadores e prefeitos e, em algumas situações, asseguradas pelo judiciário ${ }^{14}$. Mais recentemente, alguns municípios flexibilizaram as restrições antes impostas para o distanciamento social, mas tiveram de recuar em função do aumento do número de casos. Essas incertezas podem trazer consequências sérias na percepção da população, levando-a a relativizar a importância da adesão às medidas de prevenção.

Os sintomas associados à infecção pelo coronavírus 2 são mais graves em pessoas mais velhas e entre aquelas com doenças crônicas preexistentes, resultando em maiores taxas de hospitalizações, internações em Unidades de Terapia e ocorrência do óbito 3,14,15. No Brasil, até o início de junho, 86\% dos óbitos por COVID-19 haviam ocorrido entre pessoas com 50 anos ou mais 15 . Segundo o Instituto Brasileiro de Geografia e Estatística (IBGE. https://sidra.ibge.gov.br/tabela/5918, acessado em 20/ Jun/2020), estimativas para o ano de 2020 mostram que um quarto da população brasileira tem 50 anos ou mais, ou seja 54 milhões de pessoas, equivalendo à população inteira da Itália, e 30 milhões têm 60 ou mais anos de idade. Esses números dão a dimensão dos desafios a serem enfrentados pela sociedade e pelos serviços de saúde, caso a epidemia continue a evoluir ou surjam novas ondas após uma aparente calmaria.

Ao nosso conhecimento, não existem pesquisas brasileiras, com representatividade nacional, especificamente delineadas para examinar os comportamentos das faixas etárias mais velhas, em tempos da epidemia do coronavírus 2. A iniciativa ELSI-COVID-19, por meio de inquéritos telefônicos conduzidos entre os participantes do Estudo Longitudinal da Saúde dos Idosos Brasileiros (ELSI-Brasil) 16 , visa a preencher essa lacuna. Nesta publicação apresentamos os resultados da primeira série dos inquéritos telefônicos da iniciativa ELSI-COVID-19, com o objetivo de estimar as prevalências de comportamentos preventivos (distanciamento social, uso de máscaras e higienização das mãos) e examinar a associação entre este distanciamento e características sociodemográficas, tais como idade, sexo, escolaridade, situação conjugal, arranjos domiciliares e área de residência. Um objetivo adicional das nossas análises foi examinar a associação entre o distanciamento social e a presença de sintomas relacionados à COVID-19. 


\section{Métodos}

\section{Delineamento do ELSI-Brasil}

O ELSI-Brasil é um estudo longitudinal de base domiciliar, conduzido em amostra nacional representativa da população brasileira com 50 anos ou mais. Para representar a população na faixa etária elegível, o delineamento da amostra foi baseado em estágios de seleção, compreendendo municípios (unidades primárias), setores censitários e domicílios A pesquisa é conduzida em 70 municípios, situados nas cinco grandes regiões do país. O inquérito da linha de base da coorte foi conduzido em 2015-2016, com 9.412 participantes. O inquérito da segunda onda iniciou em agosto de 2019 e foi interrompido em 17 de março de 2020, em função da epidemia da SARS-CoV-2. A interrupção do trabalho de campo foi guiada por aspectos éticos, para evitar o risco potencial de transmissão durante a visita domiciliar, uma vez que a população da coorte é constituída por pessoas mais velhas. Até a interrupção do campo, 9.177 pessoas haviam sido entrevistadas e tiveram suas medidas físicas realizadas. Maiores detalhes sobre a metodologia do ELSI-Brasil podem ser vistos em Lima-Costa et al. 16 e na homepage da pesquisa (http://elsi.cpqrr.fiocruz.br/).

Todos os participantes da $2 a$ onda do ELSI-Brasil foram elegíveis para o inquérito telefônico. A entrevista telefônica foi planejada para ser curta, durando cerca de 5 minutos. Entre os elegíveis, 6.149 (67\%) responderam à entrevista. As características dos participantes do inquérito telefônico foram semelhantes às da população brasileira com 50 anos ou mais em relação a todas as características sociodemográficas analisadas, tais como idade, sexo, número de moradores no domicilio, escolaridade, região de residência e residência urbana/rural. Maiores detalhes acerca da metodologia da entrevista telefônica e razões para não respostas à mesma podem ser vistos em Lima-Costa et al. 17.

\section{Variáveis do estudo}

Na presente análise, foram consideradas as seguintes informações obtidas por meio da entrevista telefônica: frequência com que saiu de casa nos últimos 7 dias (não saiu, saiu entre 1 e 2 vezes, entre 3 e 5 vezes e quase todos os dias); razões para ter saído de casa quando este era o caso (para trabalhar, para comprar alimentos ou remédios, para encontrar amigos ou familiares, para obter atendimento de saúde, para fazer exercícios ou caminhadas, para pagar contas, por outra razão); frequência do uso de máscaras faciais quando saiu de casa (sempre, às vezes ou nunca); frequência na higienização das mãos com água/sabão ou álcool gel (sempre, às vezes ou nunca) quando saiu e retornou para a casa; e sintomas respiratórios nos últimos 30 dias (febre, tosse seca persistente e/ou dificuldade para respirar). Foram consideradas essenciais as seguintes razões para sair de casa: trabalhar, comprar remédios ou alimentos, atendimento de saúde e pagar contas. As razões consideradas não essenciais foram: fazer exercícios ou caminhada, encontrar amigos ou familiares, e outras razões não especificadas. Aqueles que informaram razões para sair de casa que se enquadravam nos dois grupos foram considerados como ter saído para atividades essenciais. $\mathrm{O}$ distanciamento social foi definido pelo relato de não ter saído de casa no período em questão.

As variáveis exploratórias incluíram informações obtidas no inquérito da 2a onda do ELSI-Brasil, a saber: idade, sexo, situação conjugal, escolaridade, número de moradores no domicilio, região de residência e situação do domicilio (rural ou urbana).

\section{Análise estatística}

Todos os resultados foram descritos em porcentagens e seus respectivos intervalos de $95 \%$ de confiança (IC95\%). Na análise não ajustada, o teste do qui-quadrado com correção de Rao-Scott foi utilizado para examinar a significância estatística das diferenças entre proporções. Modelos mutuamente ajustados, estimados por meio da regressão logística multinomial, foram usados para examinar as associações entre distanciamento social (saiu para atividades essenciais e saiu para outras atividades vs. não saiu) e as variáveis que, na análise univariada, mostraram associações com este evento em nível inferior a 0,20 . Todas as variáveis que entraram na análise multivariada foram retidas no modelo 
final. A regressão multinomial também foi utilizada para examinar como as razões para sair de casa comportam-se nas diferentes idades entre homens e mulheres, e os resultados foram ilustrados por meio de gráficos.

Todas as análises foram realizadas considerando-se o efeito do delineamento da amostra e os pesos especificamente derivados para os respondentes à entrevista telefônica. Esses pesos derivados foram estimados com base na idade, sexo e nível de escolaridade dos respondentes a essa entrevista, conforme descrito em Lima-Costa et al. 17. As análises foram realizadas usando-se o pacote estatístico Stata, versão 14 (https://www.stata.com).

O ELSI-Brasil e a iniciativa ELSI-COVID-19 foram aprovados pelo Conselho de Ética da Fundação Oswaldo Cruz (Fiocruz), Minas Gerais (CAAE: 34649814.3.0000.5091 e CAAE: 33492820.3.0000.5091, respectivamente).

\section{Resultados}

Entre os 6.149 participantes da entrevista telefônica, 6.123 responderam às perguntas acerca de comportamentos preventivos e foram incluídos nesta análise. Entre os participantes, a média da idade foi de 63,4 anos (erro padrão = 0,54), 53,4\% eram mulheres, 59,4\% eram casados e 23,3\% residiam sós. A residência na Região Sudeste predominou amplamente (40,7\%), assim como a residência em área urbana $(87,4 \%)$. Nos 30 dias precedentes, $10,4 \%$ haviam tido pelo menos um entre os seguintes sintomas gripais: febre, tosse seca persistente e/ou dificuldade para respirar.

Como pode ser visto na Tabela 1, 32,8\% dos participantes do estudo não saíram de casa nos 7 dias precedentes, $36,3 \%$ saíram entre 1 e 2 vezes, $15,2 \%$ entre 3 e 5 vezes e $15,7 \%$ saíram todos os dias. As razões para sair de casa foram as seguintes: comprar remédios ou alimentos $(74,2 \%)$, trabalhar $(25,1 \%)$, pagar contas $(24,5 \%)$, atendimento em saúde $(10,5 \%)$, encontrar familiares ou amigos $(8,8 \%)$ e fazer exercícios (6,2\%). Entre os que saíram de casa, 97,3\% usaram sempre máscaras faciais e 97,3\% sempre higienizaram as mãos. As mulheres saíram menos de casa que os homens. Em ambos os sexos, a principal razão para sair de casa foi para comprar alimentos ou remédios. Em comparação às mulheres, os homens saíram com mais frequência para trabalhar e fazer exercícios. As mulheres saíram mais para atendimento em saúde. Embora estatisticamente significantes, as diferenças por sexo no uso de máscaras e na higienização das mãos foram pouco expressivas.

$\mathrm{Na}$ Tabela 2, estão mostrados os resultados da análise não ajustada das associações entre sair de casa nos últimos 7 dias (não saiu, saiu por necessidade e saiu por outras razões), características sociodemográficas e sintomas gripais nos últimos 30 dias. Mais da metade dos participantes relatou ter saído de casa para realizar atividades essenciais, quais sejam trabalhar, fazer compras, pagar contas e busca por atendimento em saúde (56\% das mulheres e 68,1\% dos homens). As seguintes características apresentaram diferenças estatisticamente significantes $(\mathrm{p}<0,05)$ entre os grupos: faixa etária (as saídas de casa diminuíram com a idade), situação conjugal (viúvos saíram menos em comparação às demais situações), escolaridade (aqueles com maior escolaridade saíram mais de casa) e situação do domicilio (residentes em áreas urbanas saíram mais).

Na Tabela 3, estão apresentados os resultados da análise multivariada das associações entre características sociodemográficas e razões para sair de casa. As saídas de casa para as atividades essenciais foram informadas com mais frequência pelos homens $(\mathrm{OR}=1,84)$, pelos que têm escolaridade mais alta $(\mathrm{OR}=1,48$ e 1,95 para aqueles com 5-8 anos e 9 anos ou mais em comparação aos menos escolarizados, respectivamente) e pelos que residem em áreas urbanas $(\mathrm{OR}=1,54)$. As saídas para atividades essenciais foram informadas com menos frequência pelos que residiam nas regiões Centro-oeste e Norte ( $\mathrm{OR}=0,61$ e 0,54, respectivamente). As saídas para realizar atividades não essenciais foram informadas com mais frequência entre os residentes na Região Sudeste $(\mathrm{OR}=2,85$ em comparação à Região Sul) e com menos frequência entre aqueles com 70 anos ou mais $(\mathrm{OR}=0,41)$.

Nas Figuras 1 e 2, estão mostradas as probabilidades preditas das razões para sair de casa, segundo a idade como variável contínua para homens e mulheres, respectivamente. Em ambos os sexos, observa-se uma diminuição acentuada da probabilidade de ter saído de casa nos últimos 7 dias com a idade, verificando-se o oposto para saídas por razões essenciais. As saídas de casa para atividades não essenciais não foram afetadas pela idade. 
Tabela 1

Comportamentos relacionados à prevenção da infecção pelo coronavírus 2 nos últimos sete dias entre participantes do inquérito telefônico do Estudo Longitudinal da Saúde dos Idosos Brasileiros (iniciativa ELSI-COVID-19), segundo o sexo, 26 de maio a 8 de junho de 2020.

\begin{tabular}{|c|c|c|c|c|}
\hline Comportamentos & $\begin{array}{c}\text { Total } \\
\%(\text { IC } 95 \%)\end{array}$ & $\begin{array}{l}\text { Mulheres } \\
\%(I C 95 \%)\end{array}$ & $\begin{array}{l}\text { Homens } \\
\%(\text { IC } 95 \%)\end{array}$ & Valor de $p$ \\
\hline \multicolumn{5}{|l|}{ Frequência com que saiu de casa } \\
\hline Não saiu & $32,8(30,0-35,7)$ & $38,2(34,3-42,2)$ & $26,3(22,3-30,8)$ & $<0,001$ \\
\hline $1-2$ vezes & $36,3(33,3-39,4)$ & $40,1(35,1-45,3)$ & $31,9(28,3-35,6)$ & \\
\hline 3-5 vezes & $15,2(12,6-18,3)$ & $11,8(9,5-14,5)$ & $19,3(14,8-24,9)$ & \\
\hline Quase todos os dias & $15,7(12,6-19,3)$ & $10,0(7,7-12,8)$ & $22,5(17,5-28,4)$ & \\
\hline \multicolumn{5}{|l|}{ Usou máscaras faciais quando saiu de casa } \\
\hline Sempre & $97,3(96,2-98,1)$ & $98,9(98,1-99,4)$ & $95,7(93,9-97,0)$ & $<0,001$ \\
\hline Às vezes & $2,0(1,3-3,1)$ & $0,7(0,3-1,7)$ & $3,3(2,2-5,2)$ & \\
\hline Nunca & $0,7(0,4-1,2)$ & $0,4(0,2-0,5)$ & $0,9(0,4-2,1)$ & \\
\hline \multicolumn{5}{|c|}{ Higienizou as mãos com água e sabão ou álcool gel } \\
\hline Sempre & $97,3(95,7-98,4)$ & $98,6(96,6-99,4)$ & $96,1(93,5-97,7)$ & 0,038 \\
\hline Às vezes & $2,0(1,2-3,4)$ & $0,8(0,4-1,6)$ & $3,2(1,8-5,7)$ & \\
\hline Nunca & $0,7(0,2-1,9)$ & $0,7(0,1-3,7)$ & $0,7(0,2-1,9)$ & \\
\hline \multicolumn{5}{|l|}{ Razões pelas quais saiu de casa } \\
\hline Para trabalhar & $25,1(20,4-30,6)$ & $14,0(10,6-18,2)$ & $36,2(29,5-43,6)$ & $<0,001$ \\
\hline Para comprar alimentos ou remédios & $74,2(69,7-78,2)$ & $75,0(69,0-80,2)$ & $73,4(67,4-78,6)$ & 0,656 \\
\hline Para encontrar amigos ou familiares & $8,8(6,7-11,6)$ & $10,2(6,8-15,0)$ & $7,5(5,0-11,0)$ & 0,277 \\
\hline Para obter atendimento de saúde & $10,5(8,5-12,9)$ & $13,5(10,5-17,3)$ & $7,4(5,5-9,9)$ & $<0,001$ \\
\hline Para fazer exercícios ou caminhada & $6,2(4,5-8,4)$ & $3,5(2,3-5,2)$ & $8,9(6,3-12,5)$ & $<0,001$ \\
\hline Para pagar contas & $24,5(20,7-28,6)$ & $22,5(17,3-28,7)$ & $26,4(22,5-30,7)$ & 0,217 \\
\hline Por outra razão & $10,7(8,8-13,1)$ & $10,4(7,7-13,9)$ & $11,1(8,7-14,0)$ & 0,703 \\
\hline Número de entrevistados (não ponderado) & 6.123 & 3.665 & 2.458 & \\
\hline
\end{tabular}

IC95\%: intervalo de 95\% de confiança.

Valor de p: teste qui-quadrado com correção de Rao-Scott para diferenças entre os grupos.

Nota: todas as estimativas consideraram o delineamento da amostra e os pesos individuais.

\section{Discussão}

O inquérito telefônico da iniciativa ELSI-COVID-19 foi realizado entre o final de maio e o início do mês de junho, quando a epidemia estava em franca ascensão. O objetivo primário das nossas análises foi determinar a prevalência de comportamentos preventivos em relação à transmissão pelo coronavírus 2 entre adultos brasileiros mais velhos no período em questão. Os resultados mostram que somente $1 / 3$ dos participantes do estudo não havia saído de casa na semana anterior. Por outro lado, as prevalências do uso de máscara ao sair de casa e da higienização das mãos foram muito altas (cerca de 97\%). Os homens foram mais propensos a sair de casa, em comparação às mulheres, em consonância com o observado em outros contextos 18,19.

A definição de isolamento ou distanciamento social varia entre os estudos e diferentes momentos epidêmicos, tornando as comparações difíceis, tanto entre países quanto entre grupos ou indivíduos em um mesmo país. Um inquérito telefônico conduzido em Hong Kong, mostrou altas prevalências de adultos que evitavam locais com aglomeração de pessoas (de 61\% em janeiro para 85\% em março) 20. No Japão, resultados de inquérito pela internet, conduzido entre adultos no mês de fevereiro, mostrou que somente $30 \%$ dos participantes evitavam sempre e 12,6\% nunca evitavam locais com aglomeração 21. Nos Estados Unidos, um inquérito pela internet, conduzido entre março e abril, mostrou que havia grande conhecimento acerca dos sintomas e medidas de prevenção contra o coronarívus 2 e que somente $4 \%$ dos adultos haviam saído de casa nos 3 dias anteriores 18 . No Brasil, uma pesquisa 
Tabela 2

Razões para sair de casa na última semana entre participantes do inquérito telefônico do Estudo Longitudinal da Saúde dos Idosos Brasileiros, segundo características sociodemográficas e sintomas de gripe (iniciativa ELSI-COVID-19), 26 de maio a 8 de junho de 2020.

\begin{tabular}{|c|c|c|c|c|}
\hline \multirow[t]{2}{*}{ Variáveis } & \multicolumn{3}{|c|}{$\begin{array}{c}\text { Razões para sair de casa } \\
\%(\text { IC95\%) }\end{array}$} & \multirow[t]{2}{*}{ Valor de $p$} \\
\hline & Não saiu & $\begin{array}{c}\text { Saiu para atividades } \\
\text { essenciais }\end{array}$ & $\begin{array}{c}\text { Saiu para outras } \\
\text { atividades }\end{array}$ & \\
\hline \multicolumn{5}{|l|}{ Sexo } \\
\hline Feminino & $38,2(34,3-42,2)$ & $56,0(51,6-60,3)$ & $5,8(3,8-9,1)$ & 0,001 \\
\hline Masculino & $26,3(22,3-30,8)$ & $68,1(63,2-72,2)$ & $5,6(4,0-7,6)$ & \\
\hline \multicolumn{5}{|l|}{ Faixa etária (anos) } \\
\hline $50-59$ & $22,6(19,3-26,3)$ & $71,8(67,0-76,0)$ & $5,6(3,6-8,8)$ & $<0,001$ \\
\hline $60-69$ & $30,0(26,7-33,6)$ & $64,2(60,3-68,0)$ & $5,8(4,1-8,1)$ & \\
\hline 70 e mais & $56,1(50,9-61,1)$ & $38,1(33,6-42,9)$ & $5,8(4,0-8,4)$ & \\
\hline \multicolumn{5}{|l|}{ Situação conjugal } \\
\hline Solteiro(a)/Divorciado(a) & $31,1(27,4-35,2)$ & $62,2(57,0-67,2)$ & $6,7(3,5-12,5)$ & $<0,001$ \\
\hline Casado(a) & $29,1(25,8-32,7)$ & $65,6(61,5-69,5)$ & $5,3(4,0-7,0)$ & \\
\hline Viúvo(a) & $49,7(43,5-56,0)$ & $44,5(38,2-50,9)$ & $5,8(3,6-9,2)$ & \\
\hline \multicolumn{5}{|l|}{ Escolaridade (anos) } \\
\hline Até 4 & $46,4(43,1-49,9)$ & $46,6(43,4-49,8)$ & $7,0(5,5-8,8)$ & $<0,001$ \\
\hline $5-8$ & $31,4(27,4-35,7)$ & $64,3(60,1-68,2)$ & $4,3(2,9-6,6)$ & \\
\hline 9 e mais & $25,1(20,8-29,8)$ & $69,1(63,6-74,2)$ & $5,8(3,3-10,0)$ & \\
\hline \multicolumn{5}{|l|}{ Número de moradores no domicílio } \\
\hline 1 & $33,2(26,4-40,8)$ & $59,7(51,2-67,7)$ & $7,0(3,4-14,0)$ & 0,574 \\
\hline 2 & $34,0(30,4-37,7)$ & $60,0(55,8-64,0)$ & $6,1(4,4-8,4)$ & \\
\hline 3 ou mais & $31,2(26,5-36,3)$ & $64,3(58,9-69,3)$ & $4,5(3,3-6,2)$ & \\
\hline \multicolumn{5}{|l|}{ Domicílio com moradores de até 20 anos } \\
\hline Não & $32,9(29,7-36,2)$ & $60,9(57,1-64,6)$ & $6,3(4,5-8,6)$ & 0,209 \\
\hline $\operatorname{Sim}$ & $32,3(25,6-39,9)$ & $64,3(56,9-71,0)$ & $3,4(2,4-4,8)$ & \\
\hline \multicolumn{5}{|l|}{ Grande região } \\
\hline Norte & $35,7(24,7-48,6)$ & $61,3(49,1-72,2)$ & $3,0(2,2-4,1)$ & 0,092 \\
\hline Nordeste & $36,9(32,8-41,2)$ & $56,6(53,4-59,8)$ & $6,5(4,1-10,1)$ & \\
\hline Sudeste & $29,8(24,8-35,3)$ & $62,9(56,1-69,3)$ & $7,3(4,4-11,8)$ & \\
\hline Sul & $29,8(21,2-40,1)$ & $67,6(57,7-76,1)$ & $2,6(1,7-4,0)$ & \\
\hline Centro-oeste & $36,7(32,2-41,4)$ & $59,4(54,5-64,1)$ & $4,0(2,5-6,2)$ & \\
\hline \multicolumn{5}{|l|}{ Situação do domicilio } \\
\hline Urbano & $31,1(28,3-34,1)$ & $63,4(59,8-66,8)$ & $5,5(3,9-7,6)$ & 0,001 \\
\hline Rural & $44,1(37,9-50,5)$ & $48,5(43,8-53,3)$ & $7,4(4,2-12,8)$ & \\
\hline \multicolumn{5}{|l|}{ Sintomas de gripe } \\
\hline Não & $33,1(30,2-36,2)$ & $61,1(57,6-64,5)$ & $5,7(4,2-7,8)$ & 0,705 \\
\hline Sim & $29,5(20,0-41,2)$ & $65,0(52,3-76,0)$ & $5,5(2,7-10,7)$ & \\
\hline Número de entrevistados (não ponderado) & 2.532 & 3.242 & 349 & \\
\hline
\end{tabular}

IC95\%: intervalo de 95\% de confiança.

Valor de p: teste qui-quadrado com correção de Rao-Scott para diferenças entre os grupos.

Todas as estimativas consideraram o delineamento da amostra e os pesos individuais. 
Tabela 3

Análise das associações entre características sociodemográficas e razões para sair de casa na última semana, entre 6.123 participantes do inquérito telefônico do Estudo Longitudinal da Saúde dos Idosos Brasileiros (iniciativa ELSI-COVID-19), 26 de maio a 8 de junho de 2020 .

\begin{tabular}{|c|c|c|}
\hline Variáveis & $\begin{array}{c}\text { Saiu para atividades essenciais } \\
\text { OR (IC95\%) }\end{array}$ & $\begin{array}{c}\text { Saiu para outras atividades } \\
\text { OR (IC95\%) }\end{array}$ \\
\hline \multicolumn{3}{|l|}{ Sexo } \\
\hline Feminino & 1,00 & 1,00 \\
\hline Masculino & $1,84(1,37-2,46)$ * & $1,47(0,83-2,62)$ \\
\hline \multicolumn{3}{|l|}{ Faixa etária (anos) } \\
\hline $50-59$ & 1,00 & 1,00 \\
\hline $60-69$ & $0,69(0,53-0,89)$ * & $0,77(0,45-1,33)$ \\
\hline 70 e mais & $0,24(0,17-0,34)$ * & $0,41(0,25-0,67)$ * \\
\hline \multicolumn{3}{|l|}{ Situação conjugal } \\
\hline Solteiro(a)/Divorciado(a) & 1,00 & 1,00 \\
\hline Casado(a) & $1,12(0,87-1,45)$ & $0,78(0,37-1,63)$ \\
\hline Viúvo(a) & $0,94(0,63-1,39)$ & $0,79(0,46-1,37)$ \\
\hline \multicolumn{3}{|l|}{ Escolaridade (anos) } \\
\hline Até 4 & 1,00 & 1,00 \\
\hline $5-8$ & $1,48(1,12-1,94)$ * & $0,79(0,48-1,29)$ \\
\hline 9 e mais & $1,95(1,42-2,68)$ * & $1,32(0,72-2,43)$ \\
\hline \multicolumn{3}{|l|}{ Grande região } \\
\hline Sul & 1,00 & 1,00 \\
\hline Sudeste & $0,85(0,57-1,28)$ & $2,85(1,31-6,21)$ * \\
\hline Centro-oeste & $0,61(0,39-0,96)$ * & $1,23(0,57-2,62)$ \\
\hline Norte & $0,54(0,30-0,98)$ * & $0,75(0,32-1,74)$ \\
\hline Nordeste & $0,67(0,40-1,11)$ & $1,99(0,81-4,87)$ \\
\hline \multicolumn{3}{|l|}{ Situação do domicílio } \\
\hline Rural & 1,00 & 1,00 \\
\hline Urbano & $1,54(1,06-2,22)$ * & $0,87(0,44-1,75)$ \\
\hline
\end{tabular}

OR: odds ratios; IC95\%: intervalo de 95\% de confiança.

Nota: OR e IC95\% foram estimados pelo modelo de regressão multinomial e ajustados pelas variáveis listadas na tabela; não saiu de casa foi a categoria de referência. Foram consideradas essenciais as seguintes razões para sair de casa: trabalhar, comprar remédios ou alimentos, atendimento de saúde e pagar contas. As razões consideradas não essenciais foram: fazer exercícios ou caminhada, encontrar amigos ou familiares, e outras razões não especificadas. $* \mathrm{p}<0,05$ (teste de Wald).

telefônica entre adultos participantes do inquérito telefônico VIGITEL (Vigilância de Fatores de Risco e Proteção para Doenças Crônicas por Inquérito Telefônico), conduzida no mês de abril, definiu isolamento social como: ter evitado sair de casa a menos que necessário, ter evitado aglomerações de pessoas ou lugares muito cheios e ter evitado contato próximo com outras pessoas, como cumprimentos ou abraços 19 . Com base nessa definição, a prevalência do isolamento social foi de $91 \%$ 19. No Estado do Rio Grande do Sul, resultados de um inquérito de base populacional, conduzido no mês de abril, mostraram que 20,6\% dos adultos haviam saído de casa diariamente e 21,1\% haviam permanecido em casa o tempo todo; entre aqueles com 60 anos ou mais, 35,9\% não haviam saído de casa no período considerado 22 . Na presente análise, a definição de distanciamento social foi não ter saído de casa nos sete dias precedentes, observando-se uma prevalência de 33\%. Nas nossas análises, se considerarmos isolamento social como não ter saído de casa ou ter saído somente para realizar atividades essenciais, a prevalência sobe para 94\%; portanto, semelhante à do inquérito VIGITEL anteriormente mencionado 19. É interessante também observar que a prevalência de não ter saído de casa na presente análise foi muito semelhante à observada entre idosos no Rio Grande do Sul 22. 


\section{Figura 1}

Probabilidades preditas das razões para sair de casa na última semana entre os homens participantes do inquérito telefônico do Estudo Longitudinal da Saúde dos Idosos Brasileiros (iniciativa ELSI-COVID-19), segundo a idade, 26 de maio a 8 de junho de 2020.
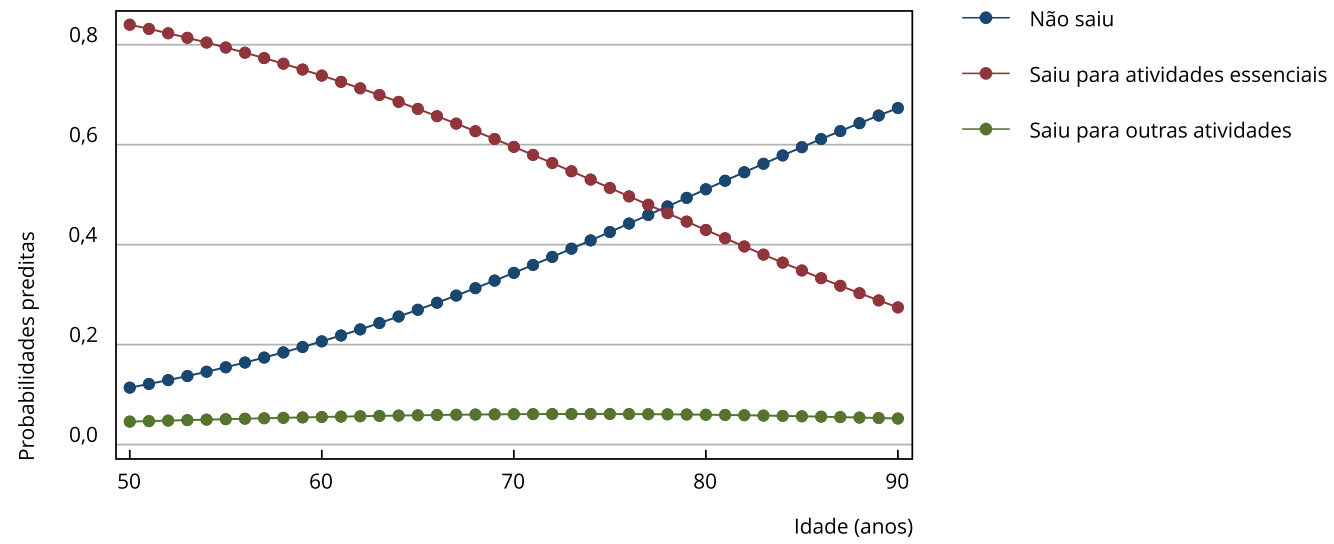

Nota: as probabilidades preditas estão ajustadas por situação conjugal, escolaridade, região de residência e residência em zona urbana/rural, conforme especificado na Tabela 3.

\section{Figura 2}

Probabilidades preditas das razões para sair de casa na última semana entre as mulheres participantes do inquérito telefônico do Estudo Longitudinal da Saúde dos Idosos Brasileiros (iniciativa ELSI-COVID-19), segundo a idade, 26 de maio a 8 de junho de 2020.

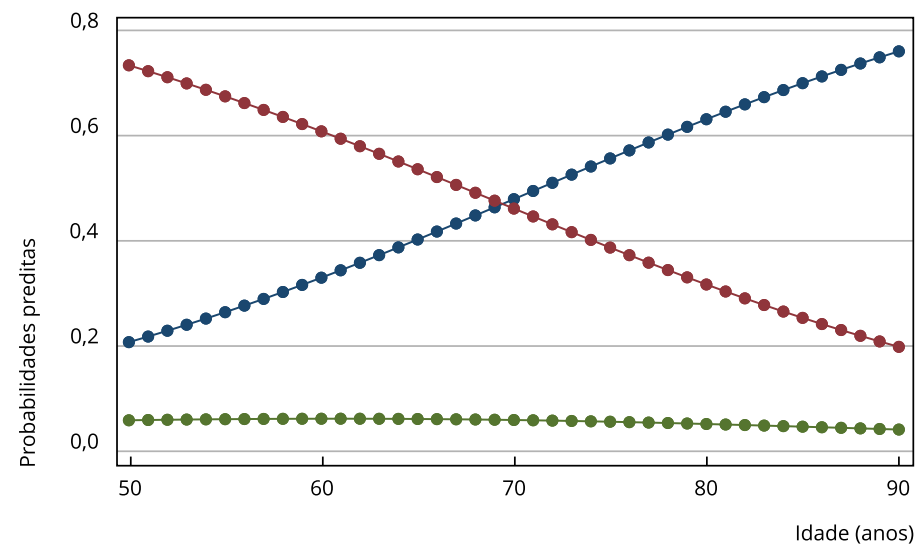

\footnotetext{
$\longrightarrow$ Não saiu

$\longrightarrow$ - Saiu para atividades essenciais

$\longrightarrow$ Saiu para outras atividades
}

Nota: as probabilidades preditas estão ajustadas por situação conjugal, escolaridade, região de residência e residência em zona urbana/rural, conforme especificado na Tabela 3. 
No Brasil, existe uma disputa de narrativas de que políticas voltadas para o distanciamento vertical, em detrimento do distanciamento horizontal (para todos), restringindo o contato com pessoas mais velhas (ou com outras vulnerabilidades), seria suficiente para conter a propagação do vírus e diminuir a mortalidade a ele associada 23 . Uma simulação matemática, baseada em dados da cidade de Belo Horizonte, Minas Gerais, comparou três cenários: nenhum distanciamento social, distanciamento vertical e distanciamento horizontal (todas as faixas etárias). Os resultados sugeriram que a adoção do distanciamento vertical é marginalmente mais efetiva para reduzir a transmissão em comparação a nenhum isolamento. Em contraste, o maior potencial de redução da transmissão é observado para o distanciamento horizontal 23. Essa simulação está de acordo com a experiência internacional, indicando a maior efetividade do isolamento horizontal em detrimento do isolamento vertical para achatamento da curva epidêmica 24,25,26,27,28.

Um dos achados mais importantes das nossas análises foi a forte associação inversa entre sair de casa e a idade. Os mais velhos de ambos os sexos foram muito mais propensos a permanecer em casa, em comparação aos mais jovens. As saídas por necessidade explicam a quase totalidade da redução dessas saídas ao longo da idade, em comparação às saídas por outras razões. Isso sugere que, com o aumento da idade, existem mais necessidades e recebimentos de ajudas de outras pessoas para a aquisição de itens básicos como alimentos e medicamentos, para evitar que os mais velhos tenham de sair de casa. Nessas circunstâncias, contatos com pessoas que trazem a infecção do ambiente externo têm maior potencial de transmissão do coronavírus 2 para os mais velhos. Esse achado é importante porque sugere que a proposta de isolamento vertical em detrimento do isolamento horizontal (para todos), além da baixa efetividade $24,25,26,27,28$, é também pouco factível.

Quando o presente inquérito foi conduzido, os maiores coeficientes de incidência de casos da COVID-19 e de óbitos, por 1 milhão de habitantes, eram observados nas regiões Norte e Nordeste do país e os mais baixos nas regiões Sul e Centro-oeste 15. Em números absolutos, a Região Sudeste ocupava a primeira posição em ocorrências de óbitos, seguida pelas regiões Nordeste e Norte do país. É importante salientar que, mesmo dentro de uma mesma região, existia (e continua existindo) uma ampla gama de variação entre os municípios 16 . Nossas análises não foram desagregadas pelo nível do município, mas alguns resultados sugerem que os comportamentos preventivos não necessariamente refletem o quadro epidêmico. Três exemplos ilustram essa situação: (1) a propensão a sair de casa para atividades essenciais foi menor tanto na Região Centro-Oeste (menos atingida) quanto na Região Norte (mais atingida); (2) a propensão a sair de casa para atividades não essenciais foi muito maior entre os residentes na Região Sudeste; (3) residentes em áreas urbanas (até então mais acometidas pela epidemia) foram mais propensos a sair de casa por necessidade em comparação aos residentes em áreas rurais.

Um achado preocupante foi a ausência de associação entre os sintomas gripais nos últimos 30 dias e o distanciamento social nos 7 dias precedentes. Mesmo que a janela temporal difira entre as duas medidas, é de se esperar que pessoas com sintomas de gripe permaneçam mais tempo em casa, em função da possibilidade de que estes sintomas sejam devidos ao coronavírus 2 . A semelhança dos sintomas com a gripe comum pode levar as pessoas a menosprezar a importância desses sintomas. No Brasil, não existe um esforço coordenado de educação e comunicação pública para indicar onde e quando as pessoas com sintomas gripais devem buscar orientação médica e faltam testes específicos para a detecção do vírus. A identificação de pessoas infectadas e o rastreamento dos seus contatos são medidas altamente recomendadas para evitar a propagação do vírus 29,30 .

Existem evidências de que a prevalência da infecção pelo coronavírus 2 no Brasil é mais alta entre aqueles com pior situação socioeconômica 31. Paradoxalmente, nós observamos uma associação inversa entre o nível de escolaridade e sair de casa, ou seja, aqueles com escolaridade intermediária e mais alta foram mais propensos a sair de casa por necessidade em relação aos menos escolarizados. Em análise suplementar (não mostrada) observamos que essa associação estava presente entre os homens com escolaridade intermediária, mas não no nível mais alto, ao passo que entre as mulheres a associação foi observada para os níveis intermediário e mais alto. É possível que pessoas com maior nível de escolaridade, embora saindo mais de casa, tenham a opção de circular em ambientes mais seguros para a transmissão da infecção como, por exemplo, o uso de transportes individuais e residência em ambientes com menos aglomeração. São necessárias futuras análises para confirmar ou não essa hipótese. 
Este estudo tem vantagens e limitações. Uma das vantagens é a possiblidade de obter informações sobre comportamentos de adultos mais velhos em relação à epidemia, sem expô-los ao risco de infecção, inerente à entrevista face a face. Outras vantagens são o grande número de participantes, residentes em diferentes municípios situados em todas as grandes regiões do país, assim como a possibilidade de utilizar uma gama de informações coletadas entre os participantes da coorte em um período imediatamente anterior ao surgimento da epidemia, permitindo comparações com aquelas obtidas durante a mesma. As limitações do estudo são aquelas inerentes às pesquisas por meio de entrevistas telefônicas. Entre elas, podemos citar o não atendimento às chamadas, a recusa em prestar informações por essa via e a inexistência de números válidos, como mostrado em outra publicação acerca da metodologia da Iniciativa ELSI-COVID-19 17. Nesta análise, para compensar pela não resposta, foram usados pesos especificamente derivados para os participantes do inquérito telefônico, mas não se pode garantir que outros fatores possam ter afetado os resultados. Outra limitação deste e de outros estudos para examinar os padrões de distanciamento social é que a epidemia evolui rapidamente, e as inferências com base nos resultados são limitadas ao período nos quais as informações foram coletadas 18. Por isso, a necessidade de estudos seriados. Análises longitudinais da iniciativa ELSI-COVID-19 permitirão examinar como os fatores pesquisados se comportarão ao longo da evolução da epidemia.

Nossos resultados levam a três principais recomendações: (1) são necessárias informações claras e oportunas para que a população perceba os riscos inerentes à epidemia e aumente sua adesão ao distanciamento social; (2) são necessárias estratégias seguras para evitar que adultos mais velhos tenham de sair de casa para adquirir itens básicos, como alimentos e medicamentos, que correspondem a cerca de 2/3 das saídas de casa; (3) são necessárias orientações claras aos pacientes com sintomas gripais, para tratamento oportuno, assim como para o rastreamento dos seus contatos com a realização de testes específicos confirmatórios. Essas medidas são a base do controle da epidemia do coronavírus 2 e devem ser adotadas urgentemente de forma consistente com a situação epidemiológica no país.

\section{Colaboradores}

M. F. Lima-Costa e J. Macinko escreveram a primeira versão do artigo. J. V. M. Mambrini realizou as análises dos dados. Todos os autores contribuíram igualmente na escrita e na revisão final do texto.

\section{Informações adicionais}

ORCID: Maria Fernanda Lima-Costa (0000-00023474-2980); Juliana Vaz de Melo Mambrini (00000002-0420-3062); Fabiola Bof de Andrade (00000002-3467-3989); Sérgio William Viana Peixoto (0000-0001-9431-2280); James Macinko (00000001-8055-5441).

\section{Agradecimentos}

O inquérito de base e a segunda onda do ELSI-Brasil foram financiados pelo DECIT/SCTI) (processos no 404965/2012-1 e 28/2017) e pela Coordenação da Saúde da Pessoa Idosa da Secretaria de Atenção à Saúde - COSAPI/DAPES/SAS (TED: 20836, 22566, 23700 e 77/2019). A iniciativa ELSI COVID-19 é financiada pelo DECIT/SCTI e pelo Conselho Nacional de Desenvolvimento Científico e Tecnológico (CNPq: processo no 403473/2020-9). M. F. Lima-Costa é bolsista IA de produtividade em pesquisa do CNPq. 


\section{Referências}

1. World Health Organization. WHO DirectorGeneral's opening remarks at the media briefing on COVID-19-11 March 2020. https:// www.who.int/dg/speeches/detail/who-director-general-s-openingremarks-at-the-mediabriefing-on-covid-19-11-march-2020 (acessado em 16/Abr/2020).

2. Ministério da Saúde. Boletim Epidemiológico 10. Situação epidemiológica da COVID-19: doença pelo coronavírus 2020. https://portalarquivos.saude.gov.br/images/pdf/2020/ April/17/2020-04-16---BE10---Boletim-doCOE-21h.pdf (acessado em 17/Abr/2020).

3. COVID-19 Dashboard by the Center for Systems Science and Engineering at Johns Hopkins University (JHU). https://coronavirus. jhu.edu/map.html (acessado em 30/Jun/2020).

4. Ferguson N, Laydon D, Nedjati Gilani G, Imai N, Ainslie K, Baguelin M, et al. Impact of nonpharmaceutical interventions (NPIs) to reduce COVID-19 mortality and healthcare demand. Imperial College COVID-19 Response Team. London: Imperial College London; 2020.

5. Flaxman S, Mishra S, Gandy A. Estimating the number of infections and the impact of nonpharmaceutical interventions on COVID-19 in 11 European countries. London: Imperial College London; 2020.

6. World Health Organization. Coronavirus disease (COVID-2019) situations reports. Geneva: World Health Organization; 2020.

7. Kupferschmidt K, Cohen J. Can China's COVID-19 strategy work elsewhere? Science 2020; 367:1061-2.

8. Mahtani KR, Heneghan C, Aronson JK. What is the evidence for social distancing during global pandemics? A rapid summary of current knowledge. Oxford COVID-19 evidence service. http://www.cebm.net/oxford-covid-19 (acessado em 10/Jun/2020).

9. Andrew D, Wiese MPH, Everson J, Grijalva CG. Social distancing measures: evidence of interruption of seasonal influenza activity and early lessons of the SARS-CoV-2 pandemic. Clin Infect Dis 2020; ciaa834.

10. Canning D, Karra M, Dayalu R, Guo M, Bloom DE. The association between age, COVID-19 symptoms, and social distancing behavior in the United States. medRxiv 2020; 23 abr. https://www.medrxiv.org/content/10.1101/2 020.04.19.20065219v1.

11. Yang C. Does hand hygiene reduce SARSCoV-2 transmission? Graefe's archive for clinical and experimental ophthalmology. Graefes Arch Clin Exp Ophthalmol 2020; [Epub ahead of print].

12. Lyu W, Wehby GL. Community use of face masks and COVID-19: evidence from a natural experiment of state mandates in the US. Health Aff (Millwood)2020; 39:1419-25.
13. Brasil. Lei no 13.979 , de 6 de fevereiro de 2020 . Dispõe sobre as medidas para enfrentamento da emergência de saúde pública de importância internacional decorrente do coronavírus responsável pelo surto de 2019. Diário Oficial da União 2020; 7 fev.

14. Aquino EML, Silveira IH, Pescarini JM, Aquino R, Souza-Filho JA, Rocha AS, et al. Medidas de distanciamento social no controle da pandemia de COVID-19: potenciais impactos e desafios no Brasil. Ciênc Saúde Colet 2020; 25 Suppl 1:2423-46.

15. Ministério da Saúde. Boletim Epidemiológico 17. Situação epidemiológica da COVID-19: doença pelo coronavírus 2020. https://www. saude.gov.br/images/pdf/2020/May/29/202005-25---BEE17---Boletim-do-COE.pdf (acessado em 06/Jun/2020).

16. Lima-Costa MF, Bof de Andrade F, Souza Jr PRB, Neri AL, Duarte YAO, Castro-Costa E, et al. The Brazilian Longitudinal Study of Aging (ELSI-Brazil): objectives and design. Am J Epidemiol 2018; 187:1345-53.

17. Lima-Costa MF, Macinko J, Andrade FB, Souza Jr. PRB, Vasconcellos MTL, Oliveira CM. ELSI-COVID-19: methodology of the telephone survey on coronavirus in the Brazilian Longitudinal Study of Aging. Cad Saúde Pública 2020; 36 Suppl 3:e00183120.

18. Alsan M, Stantcheva S, Yang D, Cutler D. Disparities in coronavirus 2019 reported incidence, knowledge, and behavior among US adults. JAMA Netw Open 2020; 3:e2012403.

19. Ministério da Saúde. Boletim Epidemiológico 11. Situação epidemiológica da COVID-19: doença pelo coronavírus 2020. https://por talarquivos.saude.gov.br/images/pdf/2020/ April/18/2020-04-17---BE11---Boletim-doCOE-21h.pdf (acessado em 17/Abr/2020).

20. Cowling BJ, Ali ST, Ng TWY, Tsang TK, Li JCM, Fong MW, et al. Impact assessment of non-pharmaceutical interventions against coronavirus disease 2019 and influenza in Hong Kong: an observational study. Lancet Public Health 2020; 5:e279-88.

21. Machida M, Nakamura I, Saito R, Nakaya T, Hanibuchi T, Takamjya T, et al. Changes in implementation of personal protective measures by ordinary Japanese Citizens: a longitudinal study from the early phase to the community transmission phase of the COVID-19 Outbreak. Int J Infect Dis 2020; 96:371-5.

22. Universidade Federal de Pelotas. EPICOVID19: Epidemiologia da COVID-19 no Rio Grande do Sul. Estudo de base populacional e validação de testes diagnósticos. https://ccs2.upel.edu.br/wp/2020/04/15/ ufpel-apresenta-resultados-do estudo-sobrecovid-19/ (acessado em 20/Jun/2020). 
23. Duczmal LH, Almeida ACL, Duczmal DB, Alves CRL, Magalhães FCO, Lima MS, et al. Vertical social distancing policy is ineffective to contain the COVID-19 pandemic. Cad Saúde Pública 2020; 36:e00084420.

24. Jarvis CI, Van Zandvoort K, Gimma A, Prem $\mathrm{K}$; CMMID COVID-19 working group; Petra Klepac P, et al. Quantifying the impact of physical distance measures on the transmission of COVID-19 in the UK. BMC Med 2020; 18:124.

25. Wu Z, McGoogan JM. Characteristics of and important lessons from the coronavirus disease 2019 (COVID-19) outbreak in China: summary of a report of 72,314 cases from the Chinese center for disease control and prevention. JAMA 2020; 323:1239-42.

26. Chudik A, Hashem Pesaran M, Rebucci A. Voluntary and mandatory social distancing: evidence on COVID-19 exposure rates from Chinese Provinces and selected countries. https:// www.nber.org/papers/w27039 (acessado em 17/Abr/2020).

27. Briscese G, Lacetera N, Macis M, Tonin M. Compliance with COVID-19 social-distancing measures in Italy: the role of expectations and duration. https://www.nber.org/papers/ w26916 (acessado em 17/Abr/2020).
28. Matrajt L, Leung T. Evaluating the effectiveness of social distancing interventions to delay or flatten the epidemic curve of coronavirus disease. Emerg Infect Dis 2020; 26:1740-8.

29. Ng Y, Li Z, Chua YX, Chaw WL, Zhao Z, Er $B$, et al. Evaluation of the effectiveness of surveillance and containment measures for the first 100 patients with COVID-19 in Singapore. MMWR Morb Mortal Wkly Rep 2020; 69:307-11.

30. Kucharski AJ, Klepac P, Conlan AJK, Kissler, SM, Tang ML, Fry H, et al. Effectiveness of isolation, testing, contact tracing, and physical distancing on reducing transmission of SARS-CoV-2 in different settings: a mathematical modelling study. Lancet Infect Dis 2020; S1473-3099(20)30457-6. [Online ahead of print].

31. Hallal P, Harwtwing F, Horta B, Victora GD, Silveira M, Struchiner C, et al. Remarkable variability in SARS-CoV-2 antibodies across Brazilian regions: nationwide serological household survey in 27 states. medRxiv 2020; 30 mai. https://www.medrxiv.org/content/10. $1101 / 2020.05 .30 .20117531 \mathrm{v} 1$. 


\section{Abstract}

The aim of the study was to examine the prevalence of social distancing, the use of face masks and hand washing when leaving home among Brazilian adults aged 50 or over. Data from 6,149 telephone interviews were used, conducted between May 26 and June 8, 2020 among participants in the Brazilian Longitudinal Study of Aging (ELSI-Brazil). Social distancing was defined by not having left home in the last seven days. Only 32.8\% of study participants did not leave home during the period considered, $36.3 \%$ left between one and two times, $15.2 \%$ between three and five times and 15.7\% left every day. The main reasons for leaving home were to buy medicine or food (74.2\%), to work (25.1\%), to pay bills (24.5\%), for health care (10.5\%), to exercise (6.2\%), and to meet family or friends (8.8\%). Among those who left home, 97.3\% always wore face masks and $97.3 \%$ always performed hand washing. Women left home less often than men. Men left home more often to work and exercise while women left home more often to seek healthcare. Men (odds ratio - OR = 1.84), those with higher education $(O R=1.48$ and 1.95 for 5-8 and 9 years, respectively) and urban residents $(O R=1.54)$ left home more frequently to perform essential activities, regardless of age or other characteristics. Results show low adherence to social distancing, but high prevalence in the reported use of face masks and hand washing.

Coronavirus; COVID-19; Social Isolation; Aging

\section{Resumen}

El objetivo del estudio fue examinar la prevalencia del distanciamiento social, uso de mascarillas e higienización de las manos al salir de casa entre adultos brasileños con 50 años o más de edad. Se utilizaron datos de 6.149 entrevistas telefónicas, realizadas entre el 26 de mayo y el 8 junio de 2020 entre los participantes del Estudio Brasileño Longitudinal del Envejecimiento (ELSI-Brasil por sus siglas en portugués). El distanciamiento social fue definido por no haber salido de casa en los últimos 7 días. Solamente un 32,8\% de los participantes del estudio no salieron de casa en el período considerado, 36,3\% salieron entre $1 y$ 2 veces, 15,2\% entre 3 a 5 veces y 15,7\% salieron todos los dias. Las principales razones para salir de casa fueron comprar medicamentos o alimentos (74,2\%), trabajar $(25,1 \%)$, pagar cuentas $(24,5 \%)$, atención en salud (10,5\%), hacer ejercicios $(6,2 \%)$ $y$ encontrar con familiares o amigos (8,8\%). Entre los que salieron de casa, un 97,3\% usaron siempre mascarillas faciales y un 97,3\% higienizaron siempre las manos. Las mujeres salieron menos de casa que los hombres. Estos salieron con más frecuencia para trabajar y para hacer ejercicio. Ellas salieron más para la atención en salud. Los hombres (odds ratio $-O R=1,84)$, los con escolaridad más alta $(O R=1,48$ y 1,95 para 5-8 y 9 años) y los residentes en áreas urbanas $(O R=1,54)$ salieron más para realizar actividades esenciales, independientemente de la edad y de otros factores relevantes. Los resultados muestran una baja adhesión al distanciamiento social, pero altas prevalencias en el uso de mascarillas e higienización de las manos.

Coronavirus; COVID-19; Máscaras; Aislamiento

Social; Envejecimiento
Recebido em 04/Jul/2020

Versão final reapresentada em 24/Jul/2020

Aprovado em 07/Ago/2020 